\title{
Utilization analysis of post-operative physical therapy for rotator cuff repair (2017)
}

\begin{abstract}
Rotator cuff injuries that result from work exposure, sport, trauma, or due to tendon degeneration with increasing age currently are preferentially repaired arthroscopically. Healing of the repaired rotator cuff often requires 6 to 12 months or longer depending on several conditions. Tear size, patient age, sex, return to work or sport participation, social and economic environment, and the rehabilitation program that includes physical therapy (PT). The cost of PT has become an issue that is largely driven by the number of PT sessions required to achieve the desired patient outcome. This is a study of the number of PT sessions necessary to achieve return-to-work status for workers comp patients that underwent arthroscopic rotator cuff repair by a single surgeon. A Medline search of the literature resulted in few studies that even mention the number of PT sessions. The usual conclusion reached is that further study is required and in the few studies that provide a PT session count there is no breakdown based on the tear characteristic or patient goals and no guidance based on Random Controlled Trials (RCTs). Presently, there is no scientifically supported PT session count that can be applied to individual patients and in particular workers. Clinicians must remain responsible for determining the extent and duration of therapy based on their knowledge of the characteristics of the rotator cuff tear, its repair, and patient' goals.
\end{abstract}

\author{
Blair Rhode \\ Orland Park Orthopedics, USA
}

Correspondence: Blair Rhode, Orland Park Orthopedics, I6450 S 104th AV Orland Park, IL 60467, USA, Tel 708-364844I, Email Blairbones@gmail.com

Received: May 01, 2018 | Published: May 18, 2018

\section{Introduction}

Workers' Compensation traces its origins back to Germany, where Chancellor Otto Von Bismarck introduced a compulsory state run accident compensation system in $1884 .{ }^{1}$ Nine states passed workers' compensation legislation in 1911, and by 1948 every state had some form of workers' comp law on the books. Workers' Compensation is a state mandated no-fault system form of insurance that ensured that workers injured at work would receive compensation without delay and without regard to fault. It is a benefit provided in exchange for mandatory relinquishment of the employee's right to sue his or her employer under the common civil law of negligence. ${ }^{2}$ The intent of the Worker's Compensation system is to achieve timely treatment of the injured worker such that the earliest return to work in an economical fashion can be achieved.

Rotator cuff pathology ranks second only to back and neck pain in frequency of occurrence in the workplace and is this second most common reason for lost time from work in manual workers. ${ }^{3}$ When a person becomes injured at work, the insurance company and employer often institute a utilization review process that has as a primary goal cost containment. Workers' Compensation is a state mandated nofault system form of insurance that ensured that workers injured at work would receive compensation without delay and without regard to fault. The injured worker wants quality medical treatment to enable a full recovery, and the insurance carrier wants as inexpensive resolution as possible.

Studies have shown that early referral to a specialist with subsequent appropriate surgical management can result in a 10 fold decrease in medical costs and a $60 \%$ decrease in time waiting for surgery and time off work. ${ }^{4}$ We have previously demonstrated that removal of barriers to care in a subset of injured workers suffering from compressive neuropathies resulted in a decrease in time to MMI from 47 months down to 5.7 months with a cost savings of greater than $\$ 200,000 .^{5}$
While the immediate cost of the clinical treatment of rotator cuff tears is significant, often it is not the most expensive aspect of RCRs. The cost of physical therapy (PT) after RCR can exceed the surgical costs. PT expense has a large range owing to the variety of RC pathologies, tear characteristics, patient age, shoulder trauma, and work demands. Cost containment pressures from insurers, employers, and payers call for reduction in outlays and may enforce limited PT. For example, Medicare specifies 21 PT sessions maximum after RCR at which point most patients will voluntarily terminate PT contact rather than personally pay for further rehabilitation. In workers compensation, insurers will often reference clinical practice guidelines through an internal utilization review process to justify limiting the number of PT sessions.

The most frequently used clinical practice guidelines are the Official Disability Guidelines (ODG) published by the for-profit Work Loss Institute. ${ }^{6}$ It states that the guideline is "based on an aggregate of over 10 million cases and decades of research, including an ongoing systematic medical literature review." In the most recent (2018) evaluation of ODG Guidelines a number of weaknesses were identified: "limited input from workers with occupational conditions, inadequate information about the process by which evidence is identified and synthesized, and inadequate documentation regarding whether the ODG chapter development teams were free of conflicts of interest and had editorial independence". 7

The ODG therapy utilization guidelines state that 24 visits of PT are appropriate after a standard arthroscopic rotator cuff repair. PT after a massive RCR may require up to 40 visits [ODG Guidelines]. Review of the literature citation for the PT subsection demonstrates that only two articles were referenced. ${ }^{8,9}$ Review of the 2004 Ejnisman study demonstrated that it analysed the effectiveness of surgical versus nonsurgical treatment for rotator cuff tears and tendinopathy. ${ }^{8}$ The study did not specifically look at PT quantification or therapy post-surgical repair. The second study was a baseball study that evaluated preseason assessment of external rotation strength and 
predicting pitchers at risk of injury. The study did not look at therapy quantification or post rotator cuff repair management.

\section{Methods}

This is a Level 3 Case series of 31 arthroscopic rotator cuff repairs (ARCRs) that were performed on worker's compensation patients in 2014-2015 with one revision repair by a single surgeon. Therapy sessions were performed in the surgeon's PT facility. The patient population consisted of 22 males and 9 females. UCLA scores, ultrasound assessment and supraspinatus isolation strength were recorded at 1,3 , and 6 months intervals. The RC tear pattern was recorded as the lengths in the anterior-posterior (AP) and mediallateral (ML) dimensions in centimeters $(\mathrm{cm})$. The RC tear area was characterized as an area $=\mathrm{AP} * \mathrm{ML}$ in $\mathrm{cm}^{2}$. Return-to-work (RTW) status was rated as the ability to perform full, medium/heavy, medium, light-medium, light duty work or none as delineated by the Department of Labor definitions. The total number of physical therapist sessions was recorded. Four patients underwent work conditioning which was not considered part of post-operative physical therapy.

To determine if there were an approved number of PT sessions for workers-comp patients in their quest to return-to-work (RTW), a hybrid overview of systematic reviews of PT for RC pathology was undertaken. Systematic reviews that included essentially the same few original studies were not considered independent. Medline and Science Direct databases were searched with 'rotator cuff' AND 'physical therapy' in either the 'title' or 'abstract' as the criteria. This criterion was intentionally broad and resulted in 237 articles. Articles were eliminated based on inappropriate focus as described in their abstract or title or any complications due to the presence of concomitant disease. Articles primarily focused either on acromioplasty or adhesive capsulitis were excluded. Articles including Articles in Press in Orthopedic journals were reviewed for ARCR and PT relevance. Reference lists were examined for RCR studies that might include the number of PT sessions. Nearly every study lacked explicit PT counts.

\section{Results}

The number of PT sessions that were required for a patient to achieve maximal medical improvement given the rotator cuff tear size is illustrated in the scatter diagram in Figure 1. PT session counts covers a range of 13 to 68 for all ARCRs with an average of 42.8 (SD $=13.7$ ).

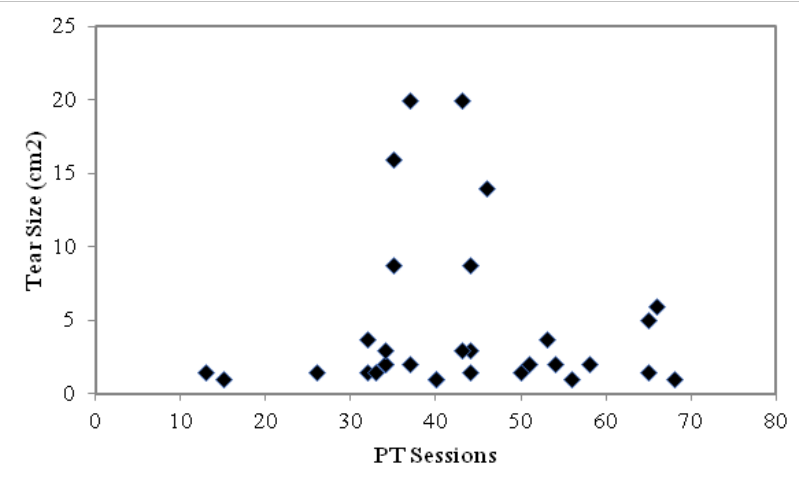

Figure 1 Scatter plot of the relationship between rotator cuff tear size and the number of physical therapy sessions to achieve final return-to-work status.
The number of PT sessions based on tear size indicates that on average fewer sessions are required when RC tears are small (Table 1), 41.5 when TS $<3 \mathrm{~cm}^{2}$ and 45.6 when TS $>3 \mathrm{~cm}^{2}$. In contrast to the ODG recommendations, large $\mathrm{RC}$ tears (massive $>3 \mathrm{~cm}^{2}$ ) did not result in significantly increased PT sessions with few requiring more than 45 sessions. Individuals that achieved RTW status required fewer sessions than those that failed to achieve RTW status. Full RTW status individuals used 35.3 sessions which was the least number for any condition (col 7), full plus medium RTW status individuals used 39.5 sessions, while those that did not achieve RTW status had 44.5 sessions. RTW status was achieved with as few as 13 sessions and as many as 68 sessions.

Table 1 Physical therapy session statistics after arthroscopic rotator cuff repair as a function of tear size and return-to-work status. PT sessions (average), Standard deviation, minimum, maximum and median number of sessions provided in bottom 5 rows. 5 th column: medium-duty and full-duty RTW status. 6th column: at best light-duty or no RTW. 7th column: workers classified as able to return to full work status.

\begin{tabular}{lllllll}
$\begin{array}{l}\text { Session } \\
\text { Statistics }\end{array}$ & $\begin{array}{l}\text { ARCR } \\
\text { all }\end{array}$ & $\begin{array}{l}\text { ARCR } \\
\text { TS }>\mathbf{3} \mathbf{c m}^{2}\end{array}$ & $\begin{array}{l}\text { ARCR } \\
\text { TS }<\mathbf{3} \mathbf{c m}^{2}\end{array}$ & $\begin{array}{l}\text { med/ } \\
\text { full } \\
\text { RTW }\end{array}$ & $\begin{array}{l}\text { No } \\
\text { RTW }\end{array}$ & $\begin{array}{l}\text { full } \\
\text { RTW }\end{array}$ \\
\hline workers & 31 & 10 & 21 & 17 & 11 & 8 \\
average & 42.8 & 45.6 & 41.5 & 39.5 & 44.5 & 35.3 \\
stdev & 13.7 & 12.2 & 14.5 & 14.1 & 12.7 & 16.8 \\
minimum & 13 & 32 & 13 & 13 & 32 & 13 \\
maximum & 68 & 66 & 68 & 66 & 68 & 58 \\
median & 43 & 43.5 & 40 & 40 & 43 & 36 \\
\hline
\end{tabular}

TS, tear size; RWT, return to work; stdev, standard deviation, ARCRs,
arthroscopic rotator cuff repairs, sessions: average PT sessions for each condition

When the RCT was $<3 \mathrm{~cm}^{2} 67 \%$ of the individuals achieved full to medium RTW status. In contrast, when the RCT was $>3 \mathrm{~cm}^{2}$ only $50 \%$ of the individuals achieved RTW status. Eight individuals (27\%) were classified as light or light-medium RTW status.

\section{Discussion}

A recent effort was made to establish evidence-based guidelines for rehabilitation after arthroscopic rotator cuff repair focused on sports injuries, a detailed rehab procedure was illustrated but without any data regarding PT sessions though active assisted range of motion was listed..$^{10} \mathrm{~A}$ few RCR studies report 12-28 PT visits after RCRs. ${ }^{11-15}$ $\mathrm{RCR}$ rehab is a progressive, integrated and a personalized process where the surgeon and physical therapist provide the patient with the information and guidance for optimum recovery. The particular surgical repair coupled with the patient's lifestyle, expectations such as return-to-work, condition of the repaired tissue, will determine the personalization of the rehabilitation program. It is likely that there is no one protocol that applies to all patients. ${ }^{12}$

The Asset (American Society of Shoulder and Elbow Therapists) organization surveyed its members who reported their average number of PT sessions per patient with 'uncomplicated' RCRs was $<25$ with a range of $12-28 .{ }^{16}$ PT visits per week varied between 1 and 4 as 'needed'. In The American Society of Shoulder and Elbow Therapists Arthroscopic Rotator Cuff Repair Rehabilitation guide the frequency and format of supervised rehabilitation four phases were described: phase 1 lasts 6 weeks with 6-12 PT sessions and 2 
visits per week for the next 20 weeks as needed. Under this protocol there would be between 46 and 52 visits for the four phases. ASSET members increase or decrease follow-up visits based on patient progress [16, Supp1]. The ASSET study allowed for differences in individual goals and needs by including a $4^{\text {th }}$ phase of rehabilitation at 20-26 weeks. ${ }^{16}$ Advanced strengthening exercises were indicated only for those patients with work or recreational demands requiring handling loads in positions not achieved by the end of phase 3 rehab. This includes patients engaging in heavy manual labor. Missing in the available data are PT session distributions based on age, sex, tear size, tissue condition, and work exposure/demands factored in.

Surgeons of the American Orthopaedic Society for Sports Medicine (AOSSM) and the Arthroscopy Association of North America (AANA) were surveyed September of 2013. ${ }^{17} 86 \%$ of surgeons reported altering rehabilitation based on tear size, $87 \%$ based on tissue quality, and $67 \%$ if there is involvement of the subscapularis tendon. It was stated that "variability in responses may in fact be a positive sign, as therapy should be individualized. The ideal postoperative physical therapy program is that which is best suited to the patient." Tear characteristics, type and quality of repair, and patient comorbidities like diabetes, smoking, and workers' compensation claim may influence rehabilitation speed.

Review of the literature demonstrated that there have been no studies performed specifically looking at postoperative physical therapy after a rotator cuff repair performed in a Worker's Compensation patient. All studies pooled their data across different patient subsets and did not delineate patients based upon age, tear type, insurance type or other con-founding variables. This is the first study to exclusively look at postoperative physical therapy and the Worker's Compensation population. Our results demonstrate that the average physical therapy visit number is 42.8 visits with significant variability $(13$ to 68 visits $(S D=13.7))$. We also demonstrated that an increase in tear size did not significantly increase the therapy count ( 41.5 when $\mathrm{TS}<3 \mathrm{~cm}^{2}$ and 45.6 when TS $>3 \mathrm{~cm}^{2}$ ).

The Official Disability Guidelines (ODG) PT Guidelines for complete rupture of the rotator cuff recommends 24 visits for a standard arthroscopic rotator cuff repair and up to 40 visits after a massive rotator cuff tear surgery. ${ }^{6}$ The ODG preface for physical therapy states "support for the physical therapy guidelines is relevant medical literature and actual experience data, combined with consensus review by experts. The most important data sources are the high quality medical studies that are referenced in the treatment guidelines, ODG Treatment in Workers' Comp, within the Procedure Summaries of each relevant chapter, summarized under the entry for Physical Therapy." The guidelines note that their treatment planning section is not designed to be a rule and as such not to be used as a basis for Utilization Review. Further, the guidelines state there is no single approach that is right for every patient.

Review of the ODG subsection for physical therapy after rotator cuff repair demonstrates that only two articles were referenced as support for the guidelines. The Ejnisman et al article looked at the evidence for the superiority of open rotator cuff repair versus arthroscopic debridement. ${ }^{8}$ The only reference to physical therapy was directed at physical therapy versus placebo in the non-operative patient. The second article by Byram assessed the correlation between preseason screening for prone external rotation strength loss and in season injury. ${ }^{9}$ Neither of these articles assessed physical therapy after surgical repair of a rotator cuff.

The insurance industry often utilizes clinical practice guidelines such as the ones sold by the Work Loss Institute as part of their utilization review process to determine the appropriateness of particular treatment regimens. Most clinical practice guidelines promote the fact that they are closely aligned with evidence base medicine. Evidencebased medicine (EBM) has been defined as the conscientious, explicit and judicious use of the best evidence for making the best decisions for patient care. Strict adherence to some set of established guidelines places the physician at risk for malpractice suits, as the standard may not be in the best interest of the individual patient.

The results of some research studies are statistically weak or incorrect, and have never been replicated by other investigators. It has been stated, "Most published research findings are false". ${ }^{18}$ An extensive line of documentation by Ioannidis and colleagues has repeatedly documented the shortcomings of clinical studies. Bias occurs as a result of design, data, analysis and presentation factors that produce findings when they shouldn't. Even when research claims are strongly contradicted by the results of randomized trials, they have been shown to persist in the literature. ${ }^{19}$ Given the time lag for development of the CPGs, they may be out of date in part or whole due to the rapidly expanding medical knowledge base. ${ }^{20}$ Only 50 percent of CPGs were found to be valid after 5.8 years.

The Work Loss Advisor Board that is in charge of creating the ODG guides is made up of 103 members from different stakeholders in the delivery of healthcare. The guidelines encompass a wide variety of medical conditions and as such the members included at least 9 chiropractors, 12 psychologists \& pain physicians, at least 20 stakeholders from insurance companies and employers. There were 10 orthopedic surgeons listed of which 4 were Emeritus (retired), 3 were spine specialists and others were in medical business or management.

Almost invariably, the peer review physician cites the ODG guidelines as their source for treatment protocols. In order for a treating physician to give an appropriate response, appropriate access to the guidelines is essential. This scientific process demands that the decision maker have access to the entire body of knowledge. This includes the availability of all of the references utilized to come to the determination. Each stakeholder involved in the decision process should also be referenced with acknowledgment of any potential bias. Any internal data or information should be made available for critical analysis. Both the ODG guides and MDguidelines reference proprietary data sets that provide the essential foundation for establishing return to work guidelines. When the data is made proprietary and not available to critical scientific analysis, the treating physician is left blind to the methods of data collection and potential bias.

Other important weaknesses to the guidelines include a lack of input from workers with occupational conditions, and limited information about the current process by which by which ODG chapter development teams identify, select, evaluate, and synthesize evidence. In addition, appraisers were uncertain whether experienced methodologists were involved in development and whether ODG chapter development teams were free of conflicts of interest and had editorial independence from the Work Loss Data Institute. ${ }^{21}$ 


\section{Conclusion}

Random Control Trials to determine the optimum number of PTs sessions for RCRs appear not to exist. Prior to this study, no research has specifically evaluated postoperative physical therapy after a rotator cuff repair exclusively in the Worker's Compensation population. We demonstrated that this specific patient population averages 43 physical therapy visits after an arthroscopic rotator cuff repair although there is significant variability. It is reasonable that the number of PT visits will vary depending on numerous factors: age, sex, health status, surgery specifics, co-morbidities, patient/athlete/worker goals, clinical evaluation, and insurance/employer requirements. No study has been able to specify the optimum number of rehabilitation visits to patient outcomes [Thigpen]. Studies of rehabilitation after RCRs have not addressed how work or sports that require significant forces and/or repetitions impact duration and rehab protocol.

Currently, final determination of PT involvement in rehabilitation must be made by the clinician as the person who knows the rotator cuff repair and understands progress resulting from rehabilitation. There remains no general consensus as to the appropriate amount of PT required to satisfy patient expectations especially when return to work (RTW) is the primary goal.

"I will apply dietetic measures for the benefit of the sick according to my ability and judgment; I will keep them from harm and injustice... Whatever houses I may visit, I will come for the benefit of the sick, remaining free of all intentional injustice." - Hippocrates, 5th century B.C.

\section{Acknowledgements}

None.

\section{Conflict of interest}

The authors have reported that they have no relationships relevant to the contents of this paper to disclose.

\section{References}

1. Guyton GP. A brief history of workers' compensation. Iowa Ortho J. 1999;19:106-110.

2. https://www.law.cornell.edu/wex/workers_compensation

3. Herberts P, Kadefors R, Högfors C, et al. Shoulder pain and heavy manual labor. Clin Ortho Relat Res. 1984;191:166-178.

4. Kemp K, Sheps D, Luciak CC, et al. Systematic review of rotator cuff tears in workers' compensation patients. Occ Med. 2011;61(8):556-562.

5. Rhode BA, Rhode WS, Rhode BJ. Removal of Barriers to Care Result in Improved Outcomes for Manual Laborers with Compressive Neuropathies. MOJ Orthop Rheumatol. 2015;2(2):00041.
6. Official Disability Guidelines ${ }^{\circledR}$ (23rd annual edition) \& ODG® Treatment in Workers' Comp (16th annual edition). See Top 6 chapters. Shoulder.

7. Shetty K, Raaen L, Khodyakov D, et al. Evaluation of the Work Loss Data Institute's Official Disability Guidelines. J Occ Environ Med.2018;60(3):e146-e151.

8. Ejnisman B, Andreoli CV, Soares BG, et al. Interventions for tears of the rotator cuff in adults. Cochrane Database Syst Rev. 2004;(1):CD002758.

9. Byram IR, Bushnell BD, Dugger K, et al. Preseason shoulder strength measurements in professional baseball pitchers: identifying players at risk for injury. Am J Sports Med. 2010;38(7):1375-1382.

10. Van der MO, Westgard $\mathrm{P}$, Chandler $\mathrm{Z}$, et al. Rehabilitation after arthroscopic rotator cuff repair; current concepts review and evidencebased guidelines. Int J Sports Phys Ther. 2012;7(2):197-218.

11. Brennan GP, Parent EC, Cleland JA. Description of clinical outcomes and postoperative utilization of physical therapy services within 4 categories of shoulder surgery. J Orthop Sports Phys Ther. 2010;40:20-29.

12. Conti M, Garofalo R, Delle Rose G, et al. Post-operative rehabilitation after surgical repair of the rotator cuff. Musculoskelet Surg. 2009;93:S55S63.

13. Flatow el, Soslowsky LJ, Ticker JB, et al. excursion of the rotator cuff under the acromion. Patterns of acromial contact. Am J Sports Med. 1994;22(6):779-788.

14. Parsons BO, Gruson KI, Chen DD, et al. Does slower rehabilitation after arthroscopic rotator cuff repair lead to long-term stiffness?. J Shoulder Elbow Surg. 2010;19:1034-1039.

15. Dickinson RN, Kuhn JE, Bergner JL, et al. A systematic review of costeffective treatment of postoperative rotator cuff repairs. J Shoulder Elbow Surg. 2017;26:915-922.

16. Thigpen CA, Shaffer MA, Gaunt BW, et al. Consensus statement. The American Society of Shoulder and Elbow Therapists' consensus statement on rehabilitation following arthroscopic rotator cuff repair. $J$ Shoulder Elbow Surg. 2016;25(4):521-535.

17. Mollison S, Shin JJ, Glogau A, et al. Postoperative Rehabilitation After Rotator Cuff Repair. A Web-Based Survey of AANA and AOSSM Members. Orthopaedic J Sports Med. 2017;5(1):1-9.

18. Ioannidis J. Why most published research findings are false. PLoS Medicine. 2005;2:696-701.

19. Tatsioni A, Boitsis NG, Ioannidis JP. Persistence of contradicted claims in the literature. JAMA. 2007;298(21):2517-2526.

20. Shekelle PG, Ortiz E, Rhodes S, et al. Validity of the Agency for Healthcare Research and Quality Clinical Practice Guidelines: How quickly do guidelines become outdated. JAMA . 2001;286(12):1461-7.

21. Nuckols TK, Shetty K, Raaen L, et al. Technical Quality and Clinical Acceptability of a Utilization Review Guideline for Occupational Conditions. ODG ${ }^{\circledR}$ Treatment Guidelines by the Work Loss Data Institute. 2017. 